\title{
New technique to determine beta half-lives in complex background conditions
}

T. Kurtukian-Nieto, ${ }^{1, a}$, J. Benlliure ${ }^{1}$, K.-H. Schmidt ${ }^{2}$, L. Audouin ${ }^{3}$, F. Becker ${ }^{2}$, B. Blank ${ }^{4}$, E. Casarejos ${ }^{1}$,

D. Cortina-Gil ${ }^{1}$, M. Fernandez-Ordoñez ${ }^{1}$, J. Giovinazzo ${ }^{4}$, D. Henzlova ${ }^{2}$, B. Jurado ${ }^{4}$, J. Pereira ${ }^{1}$,

F. Rejmund ${ }^{5}$, and O. Yordanov ${ }^{2, b}$

1 Universidad de Santiago de Compostela, 15782 Santiago de Compostela, Spain

2 Gesellschaft für Schwerionenforschung mbH (GSI), 64291 Darmstadt, Germany

3 Institut de Physique Nucléaire, 91406 Orsay Cedex, France

${ }^{4}$ CENBG, 33175 Gradignan, France

5 GANIL, Bd Henri Becquerel, BP. 55027, 14076 Caen Cedex 5, France

\begin{abstract}
Very neutron-rich nuclei near the $\mathrm{A}=195 \mathrm{r}$-process waiting point were produced as projectile fragments from a ${ }^{208} \mathrm{~Pb}$ primary beam at GSI, Darmstadt, by cold fragmentation. After in-flight separation, the fragments were implanted in an active catcher, and time correlations to the subsequent beta-decay were established. Due to the periodic operation cycles of the synchrotron, providing the primary beam, the background shows a complex time structure, which prevents applying well established analytical methods to extract the half-life information. A new mathematical analysis method has been developed, which is based on a Monte Carlo code, simulating the time sequence of implantation and beta detection according to the experimental conditions, leaving the beta lifetimes and the beta detection efficiency as free parameters. In addition, both the analysis of the experimental data and the simulation were performed in time-reversed sequence. The ratio of forward/backward time spectra contains the information of the "true" fragment-beta correlations. Half-lives were obtained from two-dimensional fits of the measured and simulated ratios of time correlations in forward- and backward-time direction by the least-squares method, being the lifetime and the beta-detection efficiency the two fitting parameters. Half-lives of 8 heavy neutron-rich nuclei approaching the r-process waiting point $\mathrm{A}=195$ have been determined.
\end{abstract}

\section{Introduction}

Secondary beams produced by projectile fragmentation offer ideal conditions for investigating the properties of exotic nuclei. Especially for neutron-rich nuclei near the $\mathrm{A}=195$ r-process waiting point, cold fragmentation [1] provides an optimum production mechanism. By in-flight separation in a magnetic spectrometer, a well-defined cocktail of a few nuclides can be prepared, and the fragments can be fully identified in $Z$ and $A$. After slowing down the fragments in a controlled way, they can be implanted in an active catcher, and their subsequent radioactive decay can be recorded. The distribution of time delays between implantation and decay carries the information on the half-life of the fragment. In the simplest case of only one nuclear species and no background, this is an exponential function. However, often the measurement is not free from background. This problem is especially severe for beta decays, which have continuous energy distributions. Analytical methods have been developed for disentangling the contributions from different sources in delayed-coincidence experiments, e.g., contaminating nuclear species, daughter decays or a constant back-ground rate.

The present paper reports on an experiment on beta-decay half-lives, performed at the FRS [2] fragment separator of GSI,

\footnotetext{
${ }^{a}$ Presenting author, e-mail: teresa.kurtukian@usc.es

${ }^{b}$ Present address: INRNE, 1784 Sofia, Bulgaria
}

Darmstadt, with a ${ }^{208} \mathrm{~Pb}$ primary beam delivered by the SIS 18 heavy-ion synchrotron. When analysing the time-delay spectra from position- and time correlations between implantations and the subsequent beta decays, we observed that the background was modulated with the periodic beam time structure of the synchrotron accelerator. Previous experiments avoided this problem either by providing a continuous beam, by choosing very short beam pulses with a suitable repetition rate, or by switching off the beam after implantation of the nuclide of interest for a period of several times the expected half-life. Unfortunately, such operation conditions are not always compatible with the technical constraints. Often they also substantially reduce the beam dose to be obtained in a given time period, and thus they may increase the statistical uncertainties. In addition, one needs to have a good estimate of the half-life in advance, which was not available in the present case due to the large uncertainties of the theoretical predictions.

Facing this experimental challenge, we have chosen the following solution: we abandoned the requirement that the data analysis is performed on the basis of an analytical description and thus gained considerably more freedom in optimizing the experimental conditions given by the accelerator operation. However, this solution required developing an analysis procedure, which copes with the rather complex time structures present in the accumulated decay-time distributions. 

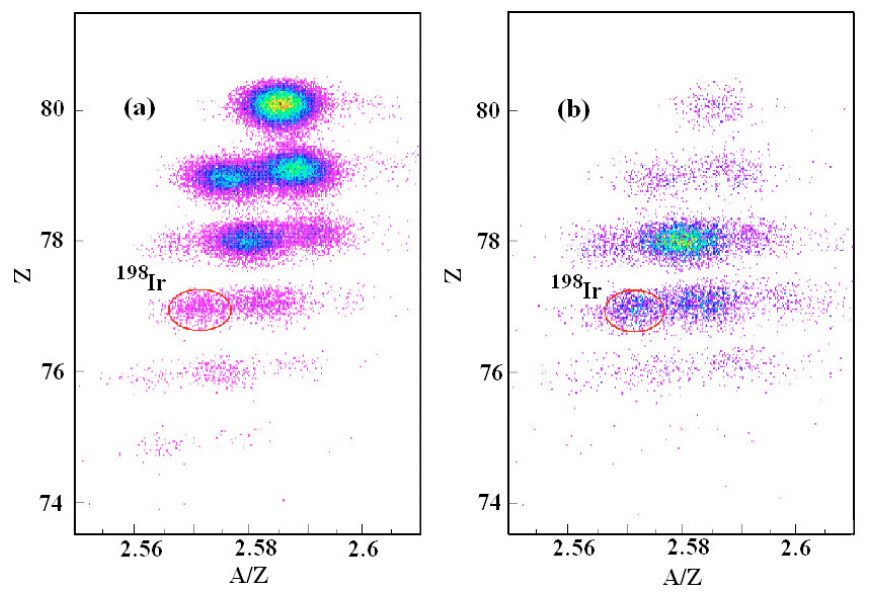

Fig. 1. Particle identification plots corresponding to a FRS setting optimised to transmit ${ }^{198} \mathrm{Ir}$ with a mono-energetic degrader. The figure shows the total yields at the exit of the FRS (a) and the ions which were implanted in the $1 \mathrm{~mm}$ thick DSSD (b).

\section{Experiment}

A beam of ${ }^{208} \mathrm{~Pb}$ at $1 \mathrm{~A} . \mathrm{GeV}$ impinged upon a beryllium target. The beam intensity was $10^{7}$ ions/s, the nominal spill length was $2 \mathrm{~s}$, and the repetition rate was $10 \mathrm{~s}$. The projectile fragments, produced in abrasion-ablation reactions, entered the fragment separator FRS. By deflection in the magnetic fields and the energy loss in a mono-energetic intermediate energy degrader [3], a number of about 9 nuclides were selected and transmitted to the final image plane. The nuclei were identified in mass and atomic number by measuring their magnetic rigidity, time-of-flight and energy loss, see figure 1 (a). In the present experiment, heavy neutron-rich isotopes from $Z=70$ up to $Z=83$ lying at and beyond the limit of the previously known nuclei have been identified. Profiting from an additional range selection, part of the produced nuclei were implanted in an active catcher, consisting of a stack of four Micron Semiconductor Ltd [4] W(DS)-1000 $5 \mathrm{~cm} \times 5 \mathrm{~cm}$ double-sided silicon strip detectors of $1 \mathrm{~mm}$ thickness. The detectors were read by 16 strips of $3.12 \mathrm{~mm}$ pitch in both the front and back side. The silicon detectors were supplemented by two scintillation detectors, in front and behind, respectively, in order to control the implantation.

Fragment implantation events were established by software, first requiring a signal above the threshold in the scintillator in front of the active catcher, no signal in the veto scintillator behind, and a high-energy signal in a given pixel of the DSSD, see figure 1 (b). The particle identification $(Z, A)$ as well as the implantation time were stored. A subsequent betalike event in the same pixel was the one that produced a signal above the threshold in a single strip in both front and back of the DSSD. Then, the beta-decay half-life was measured, correlating the delayed betas with the parent nuclei.

\section{Numerical analysis method}

Due to the complex time structure of the background, we could not use well defined analytical functions to disentangle
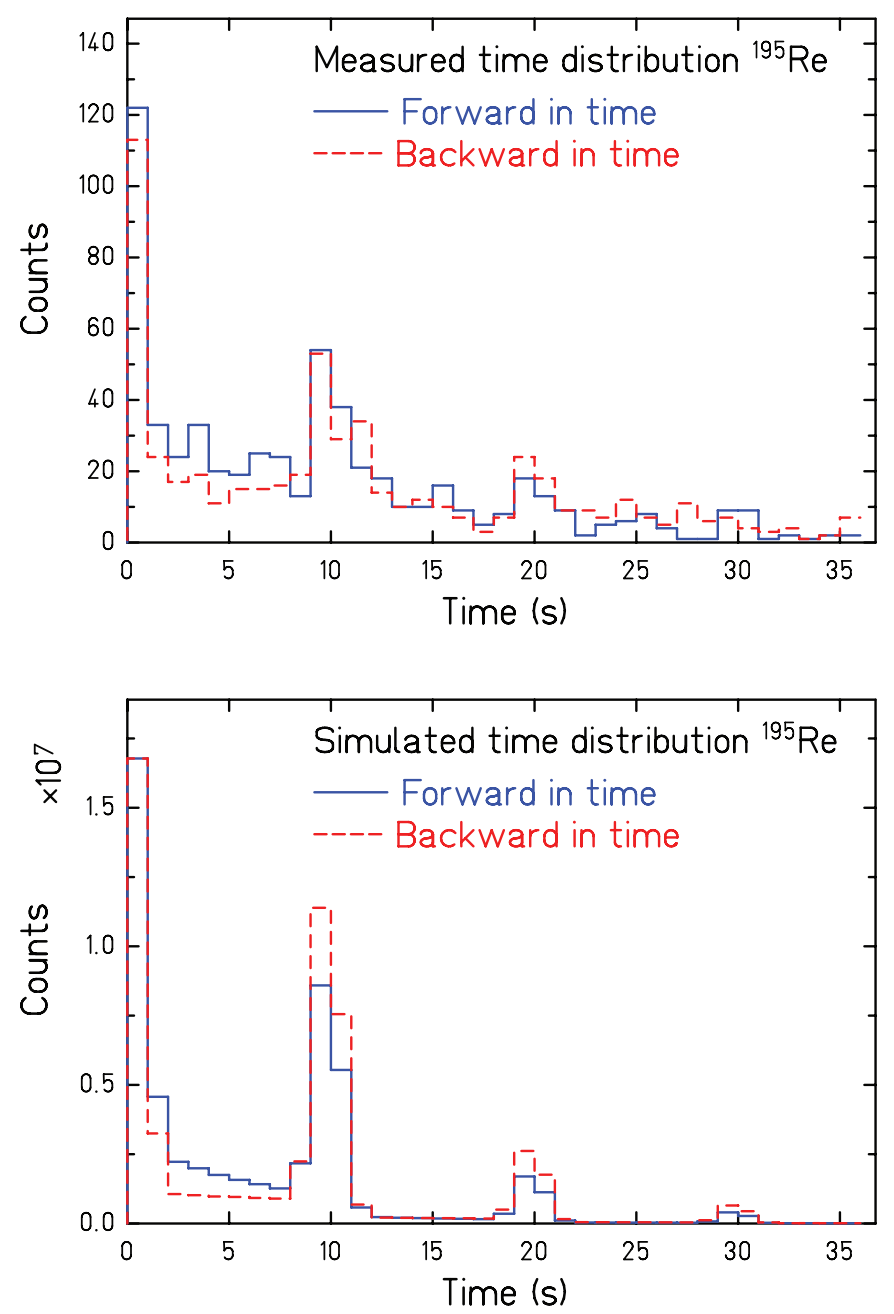

Fig. 2. Implantation-beta time correlations for ${ }^{195} \mathrm{Re}$ in forwardand backward-time sequence, accumulated during spills and pauses. Experimental data (top) are compared with a simulation, assuming $\tau=9 \mathrm{~s}$ and $\varepsilon=30 \%$ (bottom).

the true beta decays from the background events. Instead, the background was evaluated by establishing the time difference between a given implantation to a previous beta-like event. That means making the fragment-beta correlation in a timereversed sequence. We have chosen the ratio between the forward- and backward-time correlations as the signature for the "true" fragment-beta correlations.

\subsection{Monte Carlo simulation}

A Monte Carlo code has been developed to simulate the time sequences in the experiment. In this code, the time sequences of fragment implantation and beta detection were simulated according to the experimental conditions (spill sequence, fragment-implantation rate during the spill and background rate during spill and pause), leaving two free parameters; the beta lifetime $\tau$ and the beta efficiency $\varepsilon$ for the detection of the beta decays of the nuclide of interest. The code produces timecorrelation spectra in forward- and backward-time directions. A realistic simulation of the experimental conditions with different counting rates in the different cells was performed 

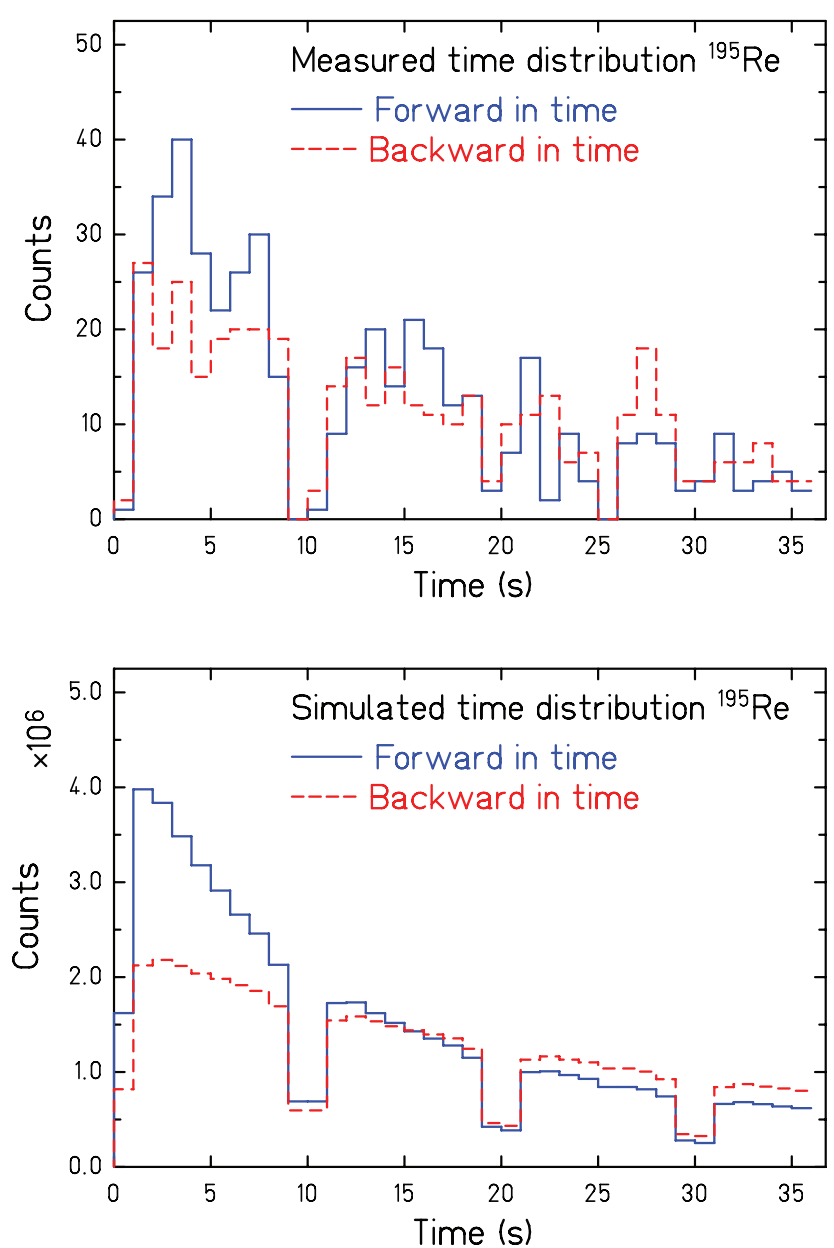

Fig. 3. Implantation-beta time correlations for ${ }^{195} \mathrm{Re}$ in forward- and backward-time sequence, accumulated only during pauses. Experimental data (top) are compared with a simulation, assuming $\tau=9 \mathrm{~s}$ and $\varepsilon=30 \%$ (bottom).

by an accumulation of a series of calculations in which the appropriate parameters for each cell were specified.

\subsection{Fitting procedure}

The rates of beta-like events found during the beam spill and in the pause between spills differ highly from one implantation setting to another, being in general much higher during the spill than in the pause. This is due to the high production rates of some species and the presence of $\delta$ electrons produced by the fast highly charged ions. As a consequence, when correlating with the first beta-like event, if the frequency of beta-like events during spill is too high, we will perform the time correlation mostly during spill, losing the information of the betas that decay during the beam pause. In order to overcome this problem, the analysis of time correlations can be performed avoiding the beam pulses, making the time correlations only in the pauses between spills.

As examples, in figures 2 and 3 we show the corresponding time-correlation spectra in forward- and backwardtime directions, establishing the time correlations of the implantation and the first consecutive beta-like event in both spill and pause and only during the pause, respectively.
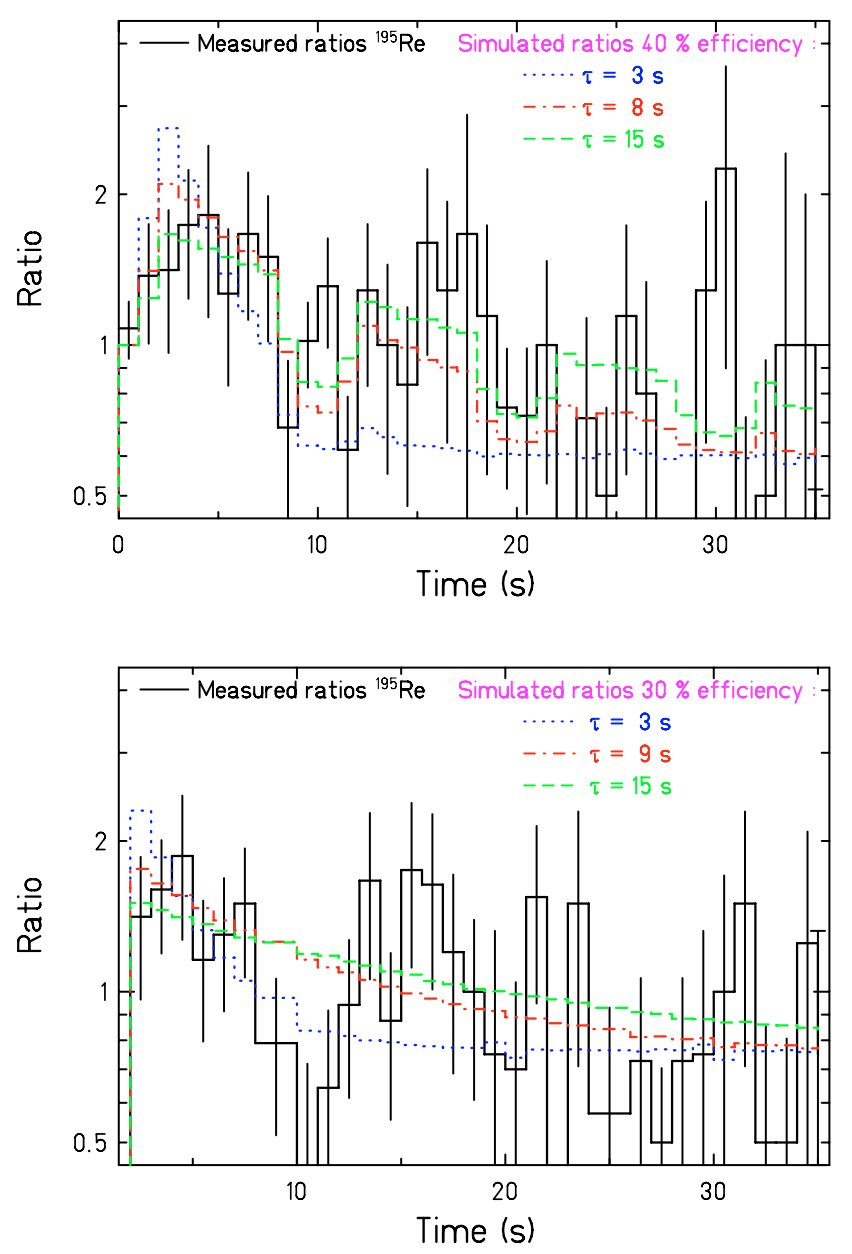

Fig. 4. Ratios of the time-difference spectra between the implantation of ${ }^{195} \mathrm{Re}$ and the first beta-like signal detected in the same strip in forward and backward time, compared with the corresponding Monte Carlo-simulated ratios for different lifetimes and a given efficiency of $40 \%$ and $30 \%$ respectively. The upper figure corresponds to the analysis extending over the full time range, while the lower figure restricts to beta-like events in the pauses only.

The experimental data are compared with the corresponding Monte Carlo simulations, which are made with much higher statistics.

The data correspond to ${ }^{195} \mathrm{Re}$, a heavy neutron-rich nuclide synthesised for the first time in this experiment. We selected this nuclide, because the experimental conditions are suited for studying the fragment-beta correlations in both options, within the full time range and only during the pauses.

Figure 4 shows the measured forward/backward ratios with the experimental error bars, and a comparison with three different Monte Carlo simulated ratios, for a fixed efficiency and different lifetimes in both simulations, only during the beam pauses and using the full time range. Note that the simulations have been performed with high statistics, imposing that the statistical uncertainties of the simulations are negligible.

The simulated ratios show most clearly the typical behaviour: the ratio starts with a value larger than one. It decreases and crosses the value of one at about $2 \cdot \tau$. At still larger 
times, the ratio decreases further due to the "shielding" of background correlations in forward time direction caused by the true betas detected before. From these graphs we learn that it is necessary to make time correlations covering several spills over several half-lives in order to distinguish between different lifetimes.

In order to determine the half-life of the selected nuclide, we performed sets of simulations with a given efficiency and lifetime, and we calculated the $\chi^{2}$ from the measured and simulated ratio of the spectra of time correlations in forwardand backward-time directions for each set of simulations. This allowed us to produce 2-dimensional $\chi^{2}$ contour plots like the ones shown in figure 5. From these contour plots we found that there is a minimum in both parameters, efficiency and lifetime, from which we can determine the half-life of the nuclide. Both types of analysis, full time and only pause, give consistent results; $T_{1 / 2}=(6 \pm 2) \mathrm{s}$ when using the full time range, and $T_{1 / 2}=(6 \pm 1) \mathrm{s}$ when correlating fragment and beta decay only between beam pulses.

In the present case, the statistical uncertainty is smaller, if the correlation analysis is restricted to the pauses. It is advantageous to avoid the large number of background events, especially during the first spill, while only few true betas are lost during this time. For nuclear species with shorter halflives, one expects the opposite. In this case, the correlation analysis should extend over the whole time range.

\subsection{Applicability of the method}

The statistical limitations of a delayed-coincidence experiment have been considered in ref. [5]. We follow this discussion and describe our experience when applying the new numerical method. Let $N_{F}$ be the total number of implanted fragments, from which we want to determine the half-life $T_{1 / 2}, v_{\beta}$ the background rate of beta-like signals, and $\varepsilon$ the beta detection efficiency. The number of "true" beta decays detected during a time equal to $T_{1 / 2}$ after the implantation of the fragment is then $\mathrm{N}_{\mathrm{F}} \cdot \varepsilon / 2$, and the beta-like background detected in the same time is $\mathrm{N}_{\mathrm{F}} \cdot v_{\beta} \cdot \mathrm{T}_{1 / 2}$. The standard deviation of the fluctuations of the random events at the same time is equal to the square root of the last expression.

From the experience we gained during the analysis we found that we can expect to obtain reliable results with our analysis method, if the number of true correlations is at least four times higher than the fluctuations of the random correlations, leading to the condition

$$
\frac{N_{\mathrm{F}} \cdot \varepsilon}{2}>4 \sqrt{N_{\mathrm{F}} \cdot v_{\beta} \cdot T_{1 / 2}}
$$

and we obtain an upper limit of the value of the half-life that we can determine:

$$
T_{1 / 2}<\frac{N_{\mathrm{F}} \cdot \varepsilon^{2}}{64 \cdot v_{\beta}} .
$$

One should consider that the value of $v_{\beta}$ is appropriately chosen: for half-lives shorter than the spill length, the value during the spill should be taken. For longer half-lives, the analysis not including the spills gives smaller statistical uncertainties, and thus the value of $v_{\beta}$ during pauses gives the more realistic estimation.
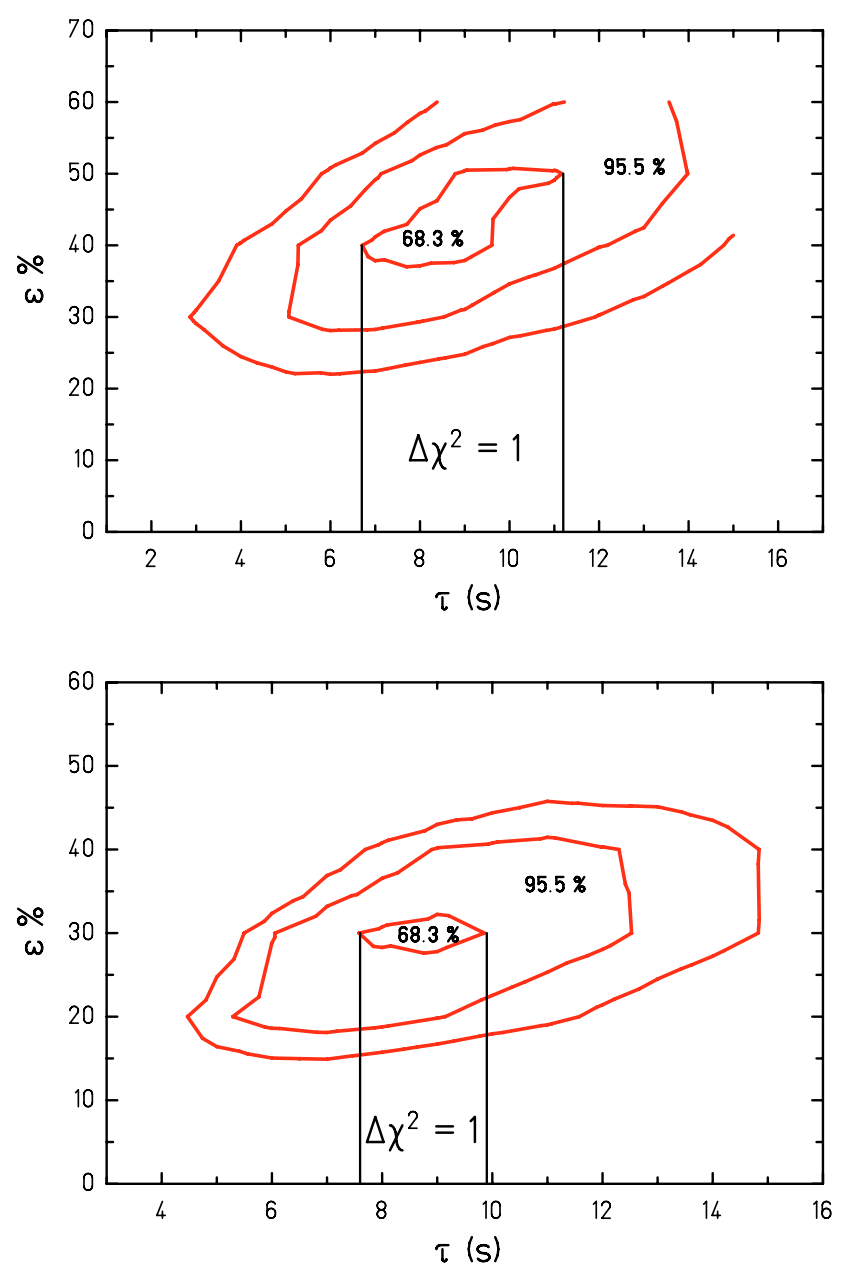

Fig. 5. Two-dimensional contour plots (lifetime-efficiency) obtained with the fitting procedure when considering the full time range (top) and only during pauses (bottom).

Table 1. Measured $\mathrm{T}_{1 / 2}$ compared with theoretical calculations.

\begin{tabular}{llll}
\hline Nuclide & $\begin{array}{l}\mathrm{T}_{1 / 2}(\mathrm{~s}) \\
\text { Exp. }\end{array}$ & $\begin{array}{l}\mathrm{T}_{1 / 2}(\mathrm{~s}) \\
\text { ref. [6] }\end{array}$ & $\begin{array}{l}\mathrm{T}_{1 / 2}(\mathrm{~s}) \\
\text { ref. [7] }\end{array}$ \\
\hline${ }^{202} \mathrm{Ir}_{125}$ & $11_{-3}^{+3}$ & 8.5 & 68.4 \\
${ }^{199} \mathrm{Ir}_{122}$ & $6_{-5}^{+3}$ & 96.6 & 370.6 \\
${ }^{198} \mathrm{Ir}_{121}$ & $8_{-2}^{+2}$ & - & 377.1 \\
${ }^{200} \mathrm{Os}_{124}$ & $6_{-3}^{+4}$ & 16 & 187.1 \\
${ }^{199} \mathrm{Os}_{123}$ & $5_{-2}^{+4}$ & 17.2 & 106.8 \\
${ }^{196} \mathrm{Re}_{121}$ & $3_{-2}^{+1}$ & 5.1 & 3.6 \\
${ }^{195} \mathrm{Re}_{120}$ & $6_{-1}^{+1}$ & 10.3 & 3.3 \\
${ }^{194} \mathrm{Re}_{119}$ & $1_{-0.5}^{+0.5}$ & 16.1 & 70.8 \\
\hline
\end{tabular}

\section{Results}

Table 1 show a summary of the experimental measurements and a comparison with the Gross-Theory calculations [6] and the hybrid-model FRDM with Gamow-Teller decays in RPA and first-forbidden (ff) decays in the Gross-Theory [7]. The experimental values deviate strongly from the theoretical calculations for most of the cases, being smaller 
than expected. The differences observed between the model calculations themselves and the measured half-lives, require further effort to improve and extend both the theoretical calculations and the experimental studies in this region.

\section{Conclusion}

We have developed a new numerical method for extracting the half-life information from delayed-coincidence experiments in the case of complex background conditions, where the conventional analysis tools, based on analytical timedistribution functions could not be applied. The method is also applicable for any other kind of complex time-dependent background. With this method, we have provided a tool which considerably extends the possibilities for exploiting the manifold advantages of the in-flight technique in determining half-lives of exotic nuclei. Beta half-lives of 8 heavy neutronrich nuclides have been measured. The shorter than expected half-lives have the astrophysical consequence of speeding up the r-process nucleosynthesis in the region near the $\mathrm{A}=195$ r-process waiting point.

\section{References}

1. J. Benlliure, K.-H. Schmidt, D. Cortina-Gil, T. Enqvist, F. Farget, A. Heinz, A.R. Junghans, J. Pereira, J. Taïeb, Nucl. Phys. A 600, 87 (1999).

2. H. Geissel et al., Nucl. Instrum. Meth. B 70, 286 (1992).

3. K.-H. Schmidt et al., Nucl. Instrum. Meth. A 260, 287 (1987).

4. http://www.micronsemiconductor.co.uk/

5. M. Bernas, P. Armbruster, J.P. Bocquet, R. Brissot, H. Faust, Ch. Kozhuharov, J.L. Sida, Z. Phys. A 336, 41 (1990).

6. T. Tachibana et al., Proc. ENAM95, Arles (1995), p. 763 http://wwwndc.tokai-sc.jaea.go.jp/CN04/index.html.

7. P. Möller et al., Phys. Rev. C 67, 055802 (2003), http://t16web/Moller/publications/rspeed2002.html. 